\title{
The Preparation of Silicon Nanowires Using Metal-Assisted Chemical Etching
}

\author{
Lu Xiaoci, Li Changqing, Ding Gangjian \\ School of Information Engineering, Zhengzhou University, Zhengzhou, China
}

Email address:

791960976@qq.com (Lu Xiaoci)

\section{To cite this article:}

Lu Xiaoci, Li Changqing, Ding Gangjian. The Preparation of Silicon Nanowires Using Metal-Assisted Chemical Etching. International Journal of Materials Science and Applications. Vol. 5, No. 3, 2016, pp. 164-168. doi: 10.11648/j.ijmsa.20160503.18

Received: June 22, 2016; Accepted: July 14, 2016; Published: July 15, 2016

\begin{abstract}
The preparation of silicon nanowires by metal catalytic chemical etching has the advantages of low cost and simple operation. This study attempts to use a silver-assisted chemical etching to prepare silicon nanowires. In the process of experiment we change the corrosive liquid concentration of corrosion and corrosion time to compare these factors on the surface topography of silicon nanowires in the preparation. The experimental results were observed by optical microscope and electron microscope. The experiment shows that in the process of etching, etching time of CLSM has had a huge impact, if etching time is short, the sample cannot generate silicon nanowires, while a long etching time will cause silicon erode up, finally dissolved in the etching liquid. The wafer surface surface morphology of honeycomb like, and the result shows that when the corrosive liquid concentration is $0.02 \mathrm{~mol} / \mathrm{L}$ and the corrosive time is four works better, formed the length $100-200 \mathrm{~nm}$ and the line diameter $100 \mu \mathrm{m}$ and more uniform silicon nanowires.
\end{abstract}

Keywords: Metal-Assisted Chemical Etching, Silicon Nanowires, SEM

\section{Introduction}

With the arrival of the electronic information age, micro electronic technology based on integrated circuit to realize the miniaturization of devices and circuits has become the basis of the information industry. But with the progress of the study, researchers have found that reduce the size of electronic devices is more and more difficult, and one dimensional semiconductor materials have great application potential in the fields of Optics and Optoelectronics because of its two dimensions has reached nanometer size, in physics, chemistry, electricity and optics, some special properties of crystalline silicon are shown [1-2], in solar cells, nano optoelectronic devices and nano electronic devices [3-5] have important application value. So it has caused the extensive concern and research. Silicon nano nanowire material preparation method can be summarized in two categories, one is top-down method, by rearrangement of atoms into nanometer materials, from the atomic or molecular starting control assembly into silicon nanowire arrays, such as the use of photolithography and etching process, by three-dimensional bulk etching of the material nanometer line; another is the bottom-up approach, such as chemical vapor deposition (CVD), thermal evaporation method, reactive ion etching (RIE).

\section{Experimental Principle}

The earliest discovery of silicon nanowires by metal catalytic chemical etching method was found by Li and Bohn [6]. The silicon substrate is coated with a thin layer of noble metal, and can be used as a catalyst for the reaction of silicon in the $\mathrm{HF}, \mathrm{H}_{2} \mathrm{O}_{2}$ and anhydrous ethanol mixed solution, and etching the vertical hole or columnar one dimensional silicon nano structure. In this paper, the preparation process is simple and the cost is low by using metal catalytic chemical etching method to prepare silicon nanowires.

The crystal to corrosion (100) mixture of $\mathrm{N}$ type silicon into silver nitrate and hydrofluoric acid in a period of time. Experimental process, the silicon wafer is processed into ultra pure water, $\mathrm{HF}$, corrosion in the mixture of ethanol and $\mathrm{H}_{2} \mathrm{O}_{2}$, silicon surface of $\mathrm{Ag}$ and $\mathrm{Si}$ in the etching solution formed a primary battery, Ag in the hole is continuously transferred to silicon, the surface silicon atom and etching liquid $\mathrm{f}$ formed $\mathrm{SiF}_{6}{ }^{2-}$ dissolved in solution. Ag contact with the $\mathrm{Si}$ is constantly dissolved, but not in contact with the Ag part of the 
formation of silicon nanowires. In the experiment, silver as electrode has a great advantage, silver will not again silicon surface formed silver film stop reaction, but the formation of diameter for nanoscale dendritic silver departure from silicon growth direction, which makes silicon wafers have been areas were exposed to the etching liquid and ensure the reaction to continue. Fig. 1 is the growth mechanism and profile of silicon nanowires.

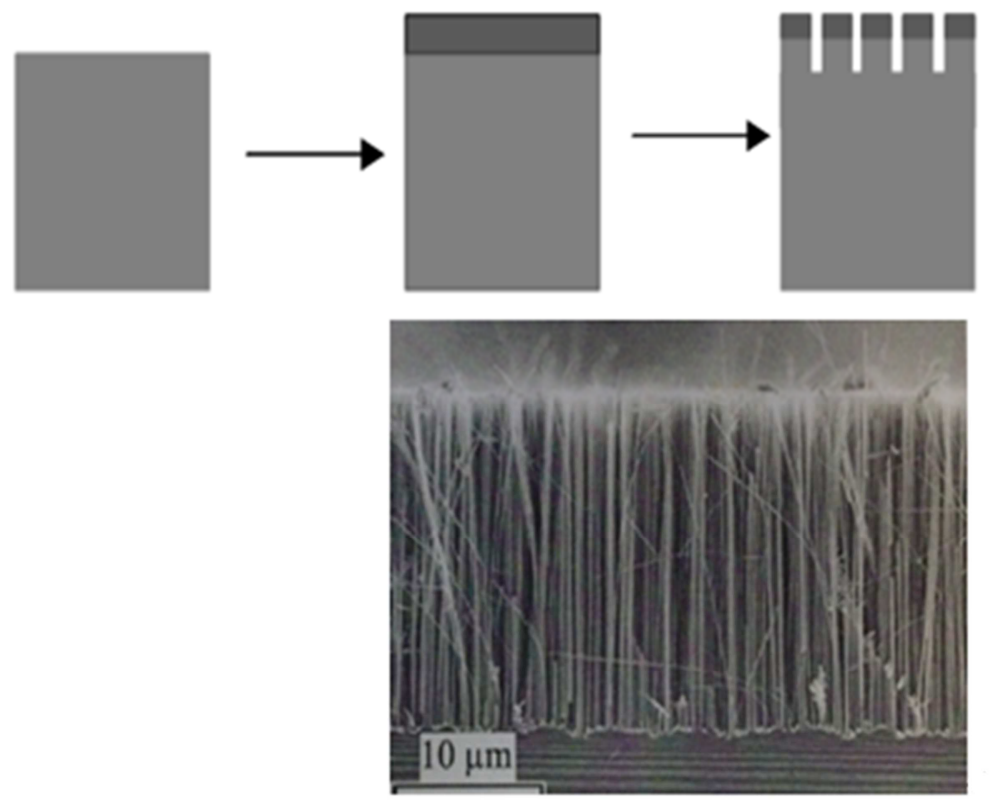

Fig. 1. Growth mechanism and profile of silicon nanowires.

In this experiment, silicon nanowires were prepared by chemical etching, and the growth mechanism was improved. In 2001, A. Splinter [7] proposed the original battery method, also known as the internal flow method. They will be plated with precious metal (generally $\mathrm{Au}, \mathrm{Ag}$ ) in the silicon into the etching solution by $\mathrm{HF}, \mathrm{H}_{2} \mathrm{O}_{2}$ and ethanol. Due to the work function of $\mathrm{Si}$ is less than the work function of the precious metal, two kinds of materials contacting, the electrons in silicon will transfer to the precious metals, and precious metal hole will transfer to the silicon, at this time, in the etching liquid form to the silicon substrate as anode, at the top of the silicon layer of precious metal as the cathode of the original battery, similar to anodic oxidation of the external current. During the experiment, the influence of these factors on the surface morphology of silicon nanowires prepared by changing the concentration of etching solution and etching time were compared.

\section{Experimental Process}

\subsection{Cleaning Before Experiment}

In the experiment, the $\mathrm{N}$ type silicon wafer with high doping $10^{16} \mathrm{~cm}^{-3}(100)$ was used as the substrate. The crystal to the (100) of highly doped n-type silicon into $0.8 * 0.5 \mathrm{~cm}^{2}$ size. As the surface of a silicon wafer cleaning degree will have serious impact on the experimental results, first of all we use standardized cleaning steps for silicon wafer cleaning. Specific methods are as follows: use the anhydrous ethanol, 5\% HF, ultra pure water cleaning, after the completion of the cleaning steps, put into the anhydrous ethanol in the stand-by.

\subsection{Etching}

Due to the use of the HF in the experiment, this experiment use PTFE beaker as a container. Configuration $\mathrm{AgNO}_{3}, \mathrm{HF}$, ethanol and hydrogen peroxide mixture, put the cleaned silicon in the etching solution, etching at room temperature for a period of time. In the experiment, the concentration of silver nitrate was $0.01 \mathrm{~mol} / \mathrm{L}, 0.02 \mathrm{~mol} / \mathrm{L}$ and $0.03 \mathrm{~mol} / \mathrm{L}$, and the etching time was $4 \mathrm{~h}, 6 \mathrm{~h}$. The experiment is divided into the following groups:

Table 1. Experimental group list.

\begin{tabular}{lllll}
\hline group & one & two & three & four \\
\hline $\begin{array}{l}\text { Concentration of } \\
\text { silver nitrate }\end{array}$ & $0.01 \mathrm{~mol} / \mathrm{L}$ & $0.02 \mathrm{~mol} / \mathrm{L}$ & $0.02 \mathrm{~mol} / \mathrm{L}$ & $0.03 \mathrm{~mol} / \mathrm{L}$ \\
Etching time & $4 \mathrm{~h}$ & $4 \mathrm{~h}$ & $6 \mathrm{~h}$ & $4 \mathrm{~h}$ \\
\hline
\end{tabular}

\subsection{Cleaning}

Take out the etched samples, clean with ultra pure water, put in a volume ratio of $\mathrm{HNO}_{3}$ 1:3 solution in $1 \mathrm{H}$, when the surface of the inclusions completely dissolution, cleaning with ultra pure water and dry by airing.

\subsection{Sample Test}

The surface morphology of the samples was observed and analyzed by CMM-55E of Shanghai chang fang optical instrument limited company and scanning electron microscope (SEM) of the Quonxe-2000 of the PHILIPS company. 


\subsection{Experimental Considerations}

(1) In the first step of etching, due to the existence of the sample surface inclusions and etched to a larger volume of air bubbles will reside in inclusions, long reaction time, generates new bubbles will enable the further increase, eventually lead to silicon and etching liquid can not contact, thereby obstructing further response; another reaction of some of the smaller bubbles can be smoothly from the inclusions in the discharge, the reaction can continue, it will cause uneven surface of a silicon wafer etching. Use the volume ratio of the removal of surface inclusions of $\mathrm{HNO}_{3}$ 1:3 solution, observed by eye wafer surface is uneven.

(2) The sample must be carefully cleaned before etching. Cleaning work is very important, if the silicon wafer surface in the cleaning process, cleaning is not clean, impurities, dirty things will on its surface, and dirty things will clamp between the metallic silver and silicon, if not be cleaned will influence the quality of preparation of silicon nanowires.

(3) Samples must be carefully cleaned and dried using ultra pure water. Due to the porous and porous structure of the metal film covered by the sample surface, a large amount of corrosion liquid can be left, and the preparation of silicon nanowires can not only influence the preparation of silicon nanowires. So after cleaning, must use the $\mathrm{PH}$ test paper to detect the cleaning solution, when the cleaning fluid is completely neutral, it proves that the cleaning completely.

(4) Most of the reagents in the experiment are corrosive, so we must pay great attention to the protective measures during the experiment. If you are not careful to contact the etching solution to the skin, should immediately rinse with plenty of water.

\section{Experimental Results Analysis}

Severe reaction at the beginning of the experiment, gray brown sponge floc formation around the wafer, sample surface is gradually Gray was sea batting shaped inclusions. When the temperature of the etching solution is at room temperature (degrees $20^{\circ} \mathrm{C}-25^{\circ} \mathrm{C}$ ), the surface of the silver layer is relatively fast, and the surface of the silicon wafer is covered with a thin layer of silver in ten minutes. As the reaction continued, there are a lot of bubbles from the surface of the sample overflow, stripped of outer layer of sponge floc samples found silver layer on the overall distribution. Figure 2 $(a, b)$ as etching after stripping the silver surface layer around the sample surface topography map, and found a silver on the sample surface layer of peripheral dendritic silver, silver wire connected to form a grid like structure, grid structure forming the etch silicon nanowires growth direction of the nanowires. In the etching process, the sample and the silver contact place is corroded, and the silver contact place formed the silicon nano wire. The surface of the sample was stripped with a few white spots on the surface of the sample, and the silver particles were observed. Silver surface layer removal found silicon surface is uneven form. This is because silicon in the etching solution with reactive ion etching, the surface of a silicon wafer with a certain degree of etching to form. And with the reaction time is different, can be observed in the sample surface in the light of the yellow, brown, dark brown and other different colors. After the reaction is completed, the sample will be taken out and washed, and the surface of the sample is dark brown, the surface of the sample is rough, and the whole is slightly thin and fragile.
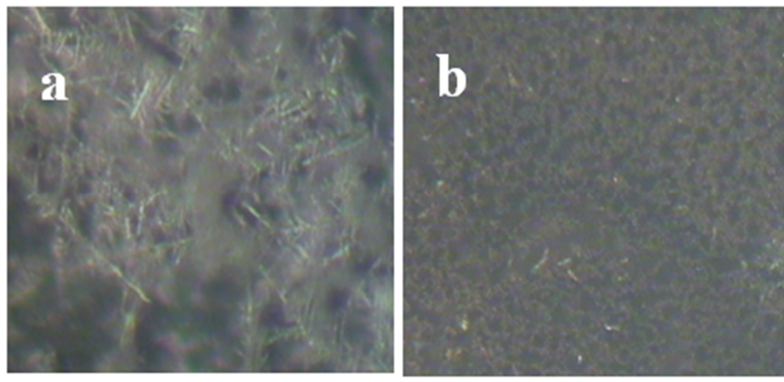

Fig. 2. Surface morphology of the samples before and after stripping.

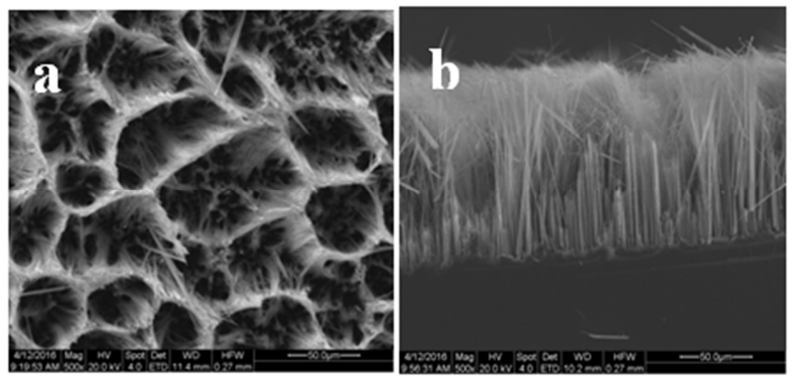

Fig. 3. The concentration of silver nitrate is $0.01 \mathrm{~mol} / \mathrm{L}$, the reaction time is $4 H$.

Figure 3 is the SEM when the concentration of silver nitrate is $0.01 \mathrm{~mol} / \mathrm{L}$ and the reaction time is $4 \mathrm{H}$. SEM samples overlooking figure (a) and a side view (b); samples SEM planform display, silicon surface formed honeycomb like morphology and some samples formed a hexagonal grid structure; side view shows that nanowires have local fracture. This may have two reasons, one is bubbles generated in the reaction of aggregation in silico, retarded the reaction speed, or even stop the reaction; another is in the initial stage of the reaction a silver similar shaped film attached to the silicon, silver surface layer did not form dendritic structure and dendritic silver, which can not form a silicon nanowires. The specific reason is not yet determined, we need to follow a series of experimental verification.
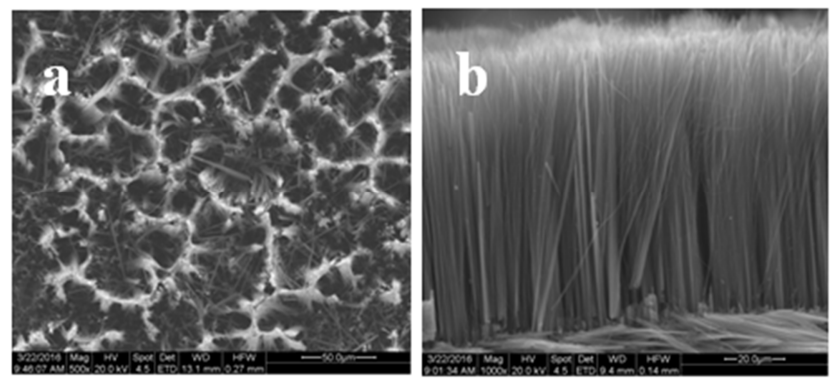

Fig. 4. The concentration of silver nitrate is $0.02 \mathrm{~mol} / \mathrm{L}$, and the reaction time is $4 H$. 
Figure 4 is the SEM when the concentration of silver nitrate is $0.02 \mathrm{~mol} / \mathrm{L}$ and the reaction time is $4 \mathrm{H}$. SEM samples overlooking figure (a) and a side view (b); by the top figure a can be seen, the sample surface silver layer was dendritic structure. When the etching time is $2 \mathrm{~h}$, the sample has a grid structure, but with the increase of etching time, the grid structure is gradually dispersed. At this time, both sides of the silicon wafer are formed by silicon nanowires, B can be seen from the side view, the formation of the silicon nanowire diameter is about $100-200 \mathrm{~nm}$, length of about $100 \mu \mathrm{m}$, arranged more neatly, and the diameter of silicon nanowires is more uniform. The sample surface can be found by electron microscope. The prepared silicon nanowires have a smaller diameter and larger length, and have a certain hardness.
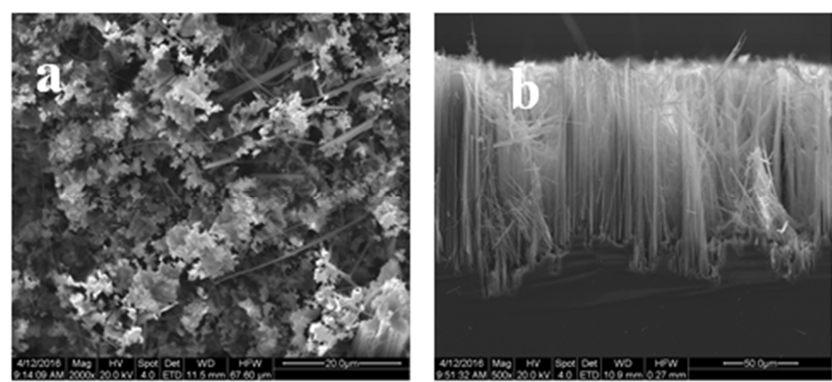

Fig. 5. The concentration of silver nitrate is $0.02 \mathrm{~mol} / \mathrm{L}$, and the reaction time is $6 H$.

Figure 5 is the SEM when the concentration of silver nitrate is $0.02 \mathrm{~mol} / \mathrm{L}$ and the reaction time is $6 \mathrm{H}$. SEM samples overlooking figure (a) and a side view (b); etching rate of silicon increases with etching time becomes slow gradually, nanometer silicon nanowire growth rate also increases with time and is slow, by that time the formation of silicon nanowires diameter of about $100 \mathrm{~nm}$ and length of about 100 $\mathrm{m}$, from which we can see the etching time is not the longer the better, because the longer the time of etching, silicon will become thinner, the preparation of silicon nanowires very prone to lodging or even break the phenomenon. If we continue etching, the surface of the silicon wafer will be etched, the sample will be dissolved from the edge, the characteristic of the sample will disappear, and finally the whole silicon wafer will be dissolved in the etching solution.

When the concentration of silver nitrate is $0.03 \mathrm{~mol} / \mathrm{L}$ and the reaction time is $6 \mathrm{H}$, the surface of a silicon wafer formed thicker layer of silver, silver closely connected, with thick silver layers, grid structure is not obvious, is not conducive to the formation of the etching process of silicon nanowires. The morphology of the samples formed by the observation of the etching is found, there are some silicon nanowires on the silicon wafer, but the diameter is large, and the arrangement is not regular.

Combined with the experimental results, the experimental conditions were selected as the concentration of silver nitrate $0.02 \mathrm{~mol} / \mathrm{L}$, reaction time of $4 \mathrm{~h}$. At this situation the prepared silicon nanowires with a smaller diameter larger length, and has a certain hardness, can better adapt to the next step of research requirements.

\section{Conclusion}

In this paper, silicon nanowires were prepared by a metal chemical etching method. The surface morphology of silicon nanowires prepared under different experimental conditions was studied. When the temperature is room temperature, compared with the silver nitrate concentration and reaction time on silicon nanowires and silver nitrate quantity is too little silver surface layer can not be formed dendritic structure, the silver layer condenses into flake, and if the amount of silver nitrate too many silver surface layer formed large area connection block, nor the formation of silicon nanowires. The surface of the silicon wafer is light yellow, yellow soil and dark brown on the surface of the silicon wafer as the etching time is prolonged. When the etching time is short, the surface of the silicon wafer has no obvious morphology. Using microscopy revealed shallow holes on the surface of a silicon wafer, wafer without obvious silicon nanowire array generation, and etching time is longer, silicon will whole is etched through, it become brittle and fragile, and etching time prolonged, silicon will starts from the edge of the dissolved until completely dissolved in solution. Experiments shows that when the amount of silver nitrate is $0.02 \mathrm{~mol} / \mathrm{L}$, the corrosion time is $4 \mathrm{~h}$, the experimental results are better, obtained samples with smaller diameter and larger length, convenient for the next experiment.

Other experiments will be carried out later, as by annealing the sample surface dendritic silver melting into net silver film, net silver film and the silicon contact more good, which the choice of the annealing temperature on the preparation of silicon nanowires looks is especially important. In addition, silicon nanowires were prepared by the addition of small current method during the second etching.

\section{References}

[1] Pei Lizhai, Tang Yuanhong, Guo Chi. Optical properties of one dimensional silicon nano materials [J]. Journal of artificial crystal, 2006 (01).

[2] Pei Lizhai, Tang Yuanhong, Guo pool. Journal of materials review [Dissertation] electrical properties of silicon nanowires, 2004 (04).

[3] Morales A M Lieber, C M. A laser [3] ablation method for the synthesis of crystalline semiconductor nanowires $[J]$. Since, 1998, 279: 208-211.

[4] Zhang Y F Tang, Y H [4], N Wang, nanowires prepared by laser ablation at high temperature [J]. Appl Phys Lett et a1. Silicon, 1998, 72 (15): 1835-1837.

[5] Tang Y H Zhang, Y F et, N Wang, a1. Morphology of Si nanowires synthesized by high temperature laser ablation [J]. J Appl Phys [5], 1999, 85 (11): 7981-7983.

[6] Li X Bohn, P W. Metal-Assited chemical in etching $\mathrm{HF} / \mathrm{H}_{2} \mathrm{O}_{2}$ produces porous silicon [J]. Appl Phys Lett [6], 2000, 77: 2572-2574.

[7] Splinter et, al. Novel porous silicon formation [7] A. technology using internal current generation Mat, Sci E C, 15 (1-2), 2001, 109-112 PP. 
[8] Canham L T. Silicon [8] quantum wire array fabrication by electrochemical and chemical dissolution of wafers [J]. Appl Phys Lett, 1990, 57: 1046.

[9] Yang Lijiao, Wang Jinliang, Yang Chengtao such as. Silicon nanowire light induced luminescence mechanism and research progress of materials [J]. Herald, 2012, 26 (21): 135-141

[10] Bai Z G Yu D $P$ Wang J J etal. Synthesis and photoluminescence properties of semiconductor nanowires $[\mathrm{J}]$. Master Sci Eng B 2000, 72: 117-120 [10].

[11] Li Changqing, Zhou Tingting, Mei Xinli, Morningstar. Chemical etching step method preparing silicon nanowires (English) [J]. Materials science and engineering of 2013 (04).

[12] Yao Rihui, Chen Ziming, impacts, Ying Cong Chen, Chen Jin Qiao. Metal catalyzed chemical etching method for preparing silicon nanowire materials [J]. Herald, 2013, 23: 1-6.

[13] Liu Li, Cao Yang, He Junhui, Yang Qiaowen. Preparation of silicon nanowire arrays and their applications in [J]. chemistry, 2013, Z1: 248-259.
[14] Zhou Yang. Study on Preparation of silicon nanowires by chemical etching [D]. Dalian University of Technology, 2011.

[15] Zhao Meihong. Preparation and optical properties of silicon nano materials [D]. Capital Normal University, 2007.

[16] Yao Rihui, Chen Ziming, impacts, Ying Cong Chen, Chen Jin Qiao. Metal catalyzed chemical etching method for preparing silicon nanowire materials [J]. Herald, 2013, 23: 1-6.

[17] Chen Ziming, impacts, Ying Cong Chen, Chen Jin Qiao, Xiao Jingyang, Yao Rihui. Based on catalytic etching of silicon nanowires preparation [J]. Functional materials. 2014, 12: 12122-12127.

[18] Zhou Jianwei, Liang Jingqiu, Liang Zhongzhu, Wang Weibiao. Optical properties of silicon nanowire arrays [J]. Journal of luminescence, 2010, 06: 894-898.

[19] Huang Yanhua, Han rang, Chen Songyan. Preparation of three-dimensional porous silicon by chemical corrosion induced by metal silver [J]. Journal of Putian University, 2015, 05: 54-59. 